\title{
$X X X$. An investigation of the potential required to maintain a current between parallel plates in a gas at low pressures
}

\section{J.A. Brown M.A.}

To cite this article: J.A. Brown M.A. (1906) XXX. An investigation of the potential required to maintain a current between parallel plates in a gas at low pressures, Philosophical Magazine Series 6, 12:69, 210-232, DOI: 10.1080/14786440609463537

To link to this article: http://dx.doi.org/10.1080/14786440609463537

曲 Published online: 16 Apr 2009.

Submit your article to this journal $\pi$

Џll Article views: 2

Q View related articles $₫$ 
position. Putting resistance in series with the condenser diminishes the magniiude of these throws very considerably.

With d'Arsonval galvanometers having unshielded coils similar effects are produced. The throws, however, are now towards the symmetrical position, and thus if the circuit be made and broken rapidly the spot of light moves to the centre of the scale whatever may be its initial position.

In conclusion the author wishes to emphasize the importance of the Rayleigh correction for the galvanometer equation. It explains many of the troublesome phenomena often noticed by those engaged in practical tests. When this correction is taken into account, the usefulness of the galvanometer is considerably increased, and the ease with which the deadpoints can be accurately determined enables this correcting factor to be readily found. The positions of the dead-points also enable us to fird the effective internal resistance of condensers. In several of the ordinary tests used in practice a knowledge of this resistance is necessary.

XXX. An Investigation of the Potential requived to maintain a Current between Parallel Plates in a Gas at Low Pressures. By J. А. Bвоw, M.A.*

\section{The Potential required to maintain a Current in a Spark-Discharge.}

IN N some recent papers Professor J. S. Townsend $\dagger$ called attention to the fact that the potential necessary to maintain a current in a gas is in general less than the potential required to cause a spark to pass, and proceeded to explain this phenomenon on the theory of collision or the production of fresh ions by the impact of positive and negative ions on the molecules of the gas. Several determinations of the current and the potential necessary to maintain it were made, using a high-resistance voltmeter and a known external resistance, which clearly showed the reality of this fact and suggested the necessity of further and more accurate measurements along these lines. Further observations were also needed with parallel plate electrodes to show definitely how the potential required to maintain a current varies with the intensity of the current, in addition to the ordinary dependence on spark-length and pressure which the law for the sparking

* Communicated by Prof. J. S. Townsend, F.R.S.

+ J. S. Townsend, Phil. Mag. viii. p. 750 (Dec. 1904); Phil. Mag. ix. p. 289 (Niarch 1905): 
potential seems to require. It was thought worth while accordingly to extend the determination of the variation of the current with the potential at various pressures, and particularly below the critical pressure.

The general phenomena of the spark-discharge are now well known The so-called Paschen's* Law, confirmed primarily by the determinations of Peacet and the careful measurements of Carr + , is now well established within certain limits. The sparking potential for parallel plate electrodes may confidently be said to depend simply on the amount of gas between the electrodes, and not to vary with the material of the plates.

A number of the phenomena in connexion with these potentials, and other effects noticed in the spark-discharge, have been explained by the theory of ionization by collision. There are peculiarities, however, which still need investigation. Under certain conditions the electrodes produce effects which cannot be explained by the simple theory mentioned above, which has so successtully accounted for the general action. When precautions are not taken to eliminate these effects, the peculiarities in the character of the discharge are very complicated; and some account of these is given in the second part of this paper.

\section{Part I.}

The direct object of the investigation, then, was the measurement of the current and at the same time the potential required to maintain it between parallel plate electrodes at comparatively small pressures.

Several similar determinations of this bave been made, notably by Riecke $\S$. All this work has, however, been undertaken with ordinary racuum-tubes, with electrodes projecting into the gas and with no attempt to secure a uniform field. In Riecke's work, for instance, the tube was a sphere, with electrodes near the ends of diameters at right angles to each other, their distance apart being approximately $6.5 \mathrm{cms}$. Sparking accordingly frequently started from the back of the cathode when the pressure was decreased below a certain value. So the statement he makes, that the value of the current and the potential to maintain it is a function depending on the tube, cannot be wondered at.

Carr has shown the necessity of trying to secure uniformity

* Paschen, Wied. Ann. xxxvii. p. 79 (1889).

† Peace, Proc. Roy. Soc. lii. p. 99 (1892).

I Garr, Proc. Roy. Soc. Ixxi. p. 374 (1903).

$\S$ Riecke, Ann. der Phys. ir. p. 592 (1901). 
of the field and definiteness of the spark-length in his discussion of Peace's results of the sparking potential. The latter for pressures below the critical value could discover no indications that Paschen's law beld true; but the precautions taken by Carr to eliminate the possibility of a brush-discharge around the edges of the plates at low pressures, and other disturbing influences, proved that the law extended to the lower pressures as well. Such precautions were accordingly also taken in the present work, in the hope that results would be more definite than any other obtained previously.

\section{Apparatus.}

The spark-gap used was of the form suggested by Carr * in his determination of sparking potentials. The only important difference was that the walls were of glass instead of ebonite, to enable one to watch the spark so as to determine whether it filled the space entirely, and whether there was any flickering or other unsteadiness.

Fig. 1.

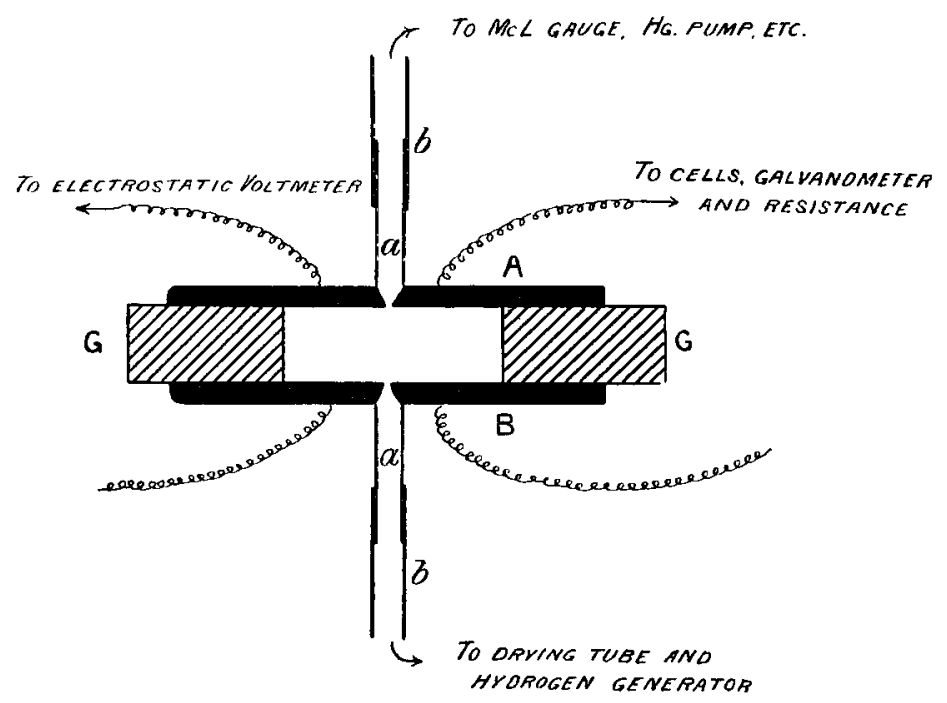

The diagram needs no lengthy explanation. The electrodes $A$ and $\mathbf{B}$ were accurately turned, three pairs in all being used, of zinc, aluminium, and silver respectively, the latter two being set into brass to enable the tubes $\alpha a$ to be more easily soldered on and to ensure a solid and air-tight plate.

The spark-chambers were connected to one another in * Carr, l.c. p. 406. 
parallel, the gas being able to flow freely from one to the other; but no difference in the results obtained from the different electrodes was eventually noticed, although at first there was a decided effect due to the material. (See Part II.)

The walls $G \mathrm{G}$ were formed by a ring of plate-glass accurately ground and polished, approximately $1 \mathrm{~cm}$. thick, the hole which formed the spark-chamber being $3 \mathrm{cms}$. in diameter. The plates were stuck to the glass with ordinary commercial elastic glue, as was also the fine brass tubing $a a$ into the glass tubes $b b$, the joints being carefully tested to see whether they were air-tight. These were the only joints made in this way, all the rest of the apparatus being glass, and the whole being frequently tested for leaks. The current was measured by an ordinary d'Arsonval galvanometer, of resistance 5.5 ohms, and giving $1 \mathrm{~mm}$. deflexion on a scale 1 metre distant for $10^{-6}$ ampere. The potential was read off on a multicellular electrostatic voltmeter, graduated up to 600 volts and connected directly to the electrodes. A battery of small storage-cells furnished the current, a large liquid resistance being always in the circuit. It was at first decided to use air in the spark-gap, but it was found that one could not be certain of steady conditions with this gas, as the spark would often flicker and suddenly become unsteady, and would not always fill the whole space. This was most probably due to an effect on the electrodes, so it was decided to use hydrogen throughout the investigation as it is so easily obtained in a pure state. The gas was prepared electrolytically from a solution of very pure $\mathrm{Ba}(\mathrm{OH})_{2}$ in a generator specially constructed to prevent any possibility of the hydrogen being contaminated by impurities. The gas, after being evolved, was carefully dried by allowing it to stand at least twenty-four hours in close contact with dry phosphoric pentoxide before being admitted to the spark-chamber. In connexion with this, too, there was a drying-tube; so it would seem that sufficient precautions were taken to ensure the gas being dry and pure.

For about four months, while the final readings were being taken, no air sufficient to be detected by the McLeod gauge came in contact with the plates at any time. To make sure of the continued purity of the gas between the plates, it was entirely cleaned out every few days, the pumping being carried down to at least $\frac{1}{80} \mathrm{~mm}$, and fresh gas was admitted. During the running of the current there was sometimes a slight absorption of gas by the electrodes, this and similar effects being noted below; but the method of taking the readings prevented any serious inaccuracy due to this cause. 


\section{Procedure.}

The method of procedure was briefly this:-'The pressure having been adjusted and carefully read on the gauge, the current was put on and readings of the galvanometer and voltmeter taken as soon as possible. Then the resistance was decreased and a new value taken. The largest current having been attained, the reverse process was followed, the resistance increased and various new points on the curve for the current determined. In this way the effect of any permanent change in the pressure could be at once detected. The curves of fig. 2 show the method of procedure.

Fig. $\supseteq$.

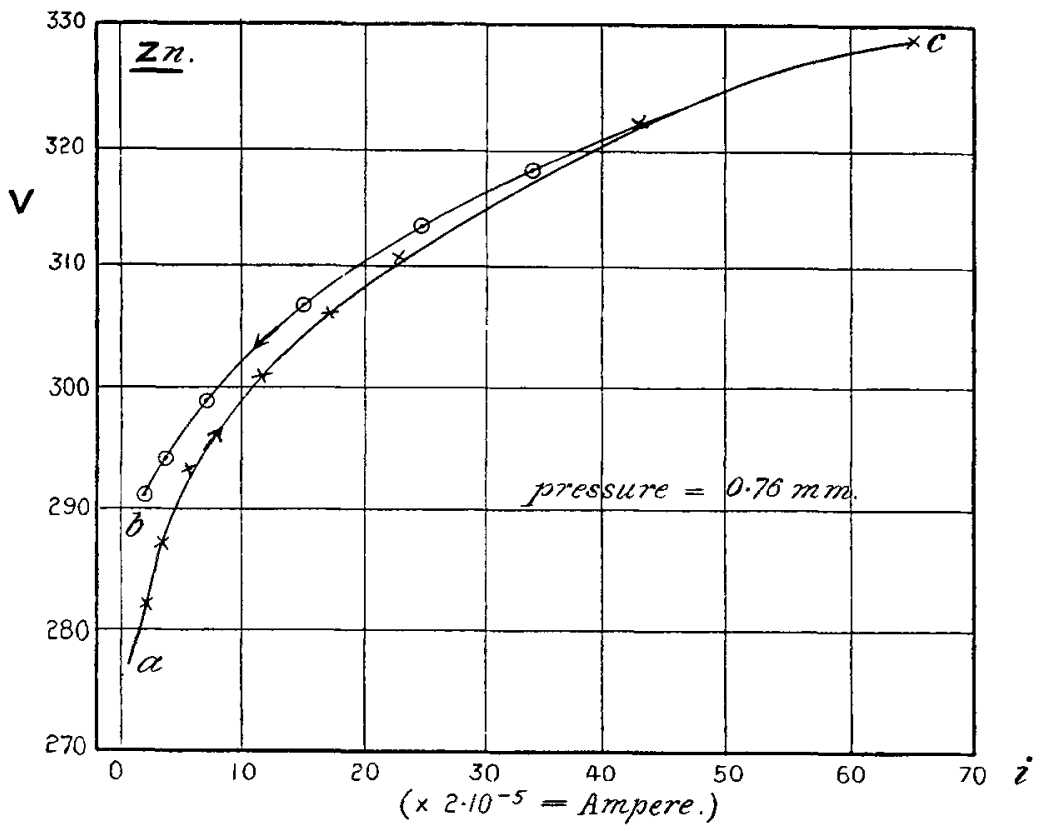

The first reading obtained was the one marked $a$ on the diagram. The resistance $\mathrm{x}$ as then gradually decreased until $c$ was reached, and then the reverse process carrie d on down to $b$. The difference in the potentials of $a$ and $b$ was so small that taking the average of these two curves would introduce no great error; and this was done, the pressure being assumed to be the mean of the readings on the gauge before and after the series. (But see later, the explanation given for the form of the curves below the critical pressure.) 
The potential of the battery was in all cases considerably in excess of the potential at the electrodes, and a large resistance was used to adjust the current, for reasons which are explained below. (See Part II. A.)

\section{Results.}

The curres for the pressures above the critical pressure (fig. 3) show clearly that the potential required to maintain a current is less than the sparking potential which is indicated on each curve (Sp. V.). It was found that the curves are the same for zinc, aluminium, or silver electrodes, those given being the average of a number obtained with zinc. The currents in the figure are expressed as amperes per square centimetre of the electrode, the distance between the plates being $1 \mathrm{~cm}$.

Fig. 3.

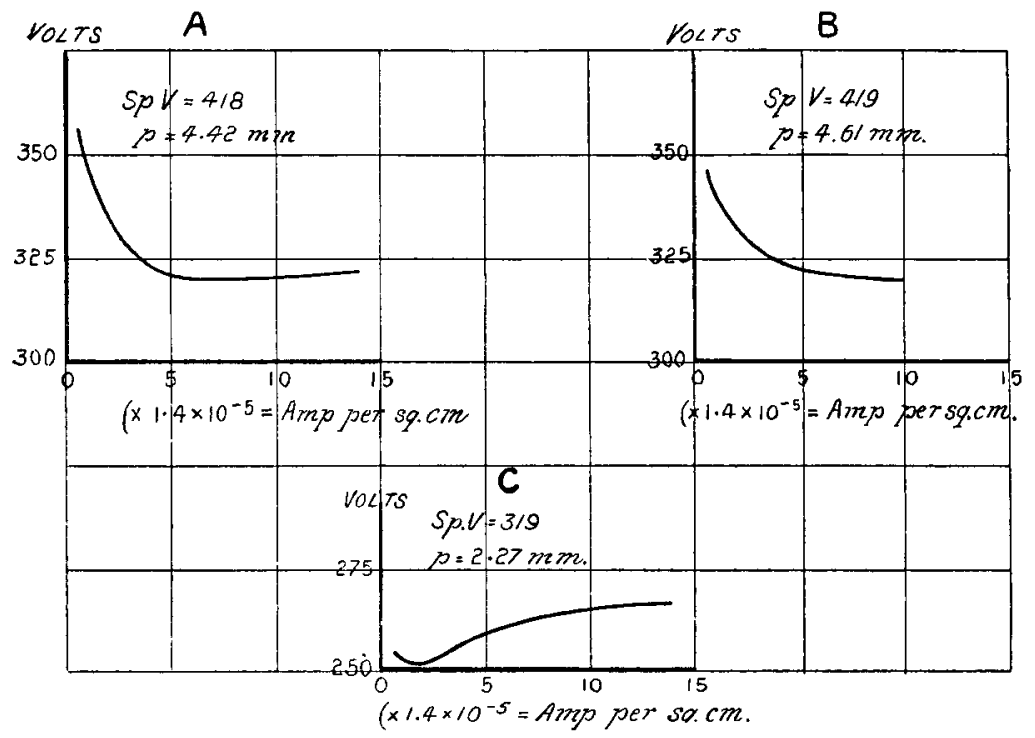

At Pressures above the Critical Pressure.

The galvanometer used was not sensitive enough to detect smaller currents than those indicated; but the tendency of the curve is shown in each case, and the sparking potential is given. This drop soon reaches a limit, and for current densities larger than $\frac{1}{10,000}$ of an ampere per sq. cm., the potential may be taken as practically constant as the current 
is increased as far as the values were taken. In general, the curves were not pushed on beyond the values given, but Curve 5, fig. 10 (see below), with aluminium plates shows that carrying the current to greater values would not introduce any change in the ahove generalization.

For pressures below the critical pressure, however the case is quite different. Curre A, fig. 4 , is practically at the

Fig. 4.

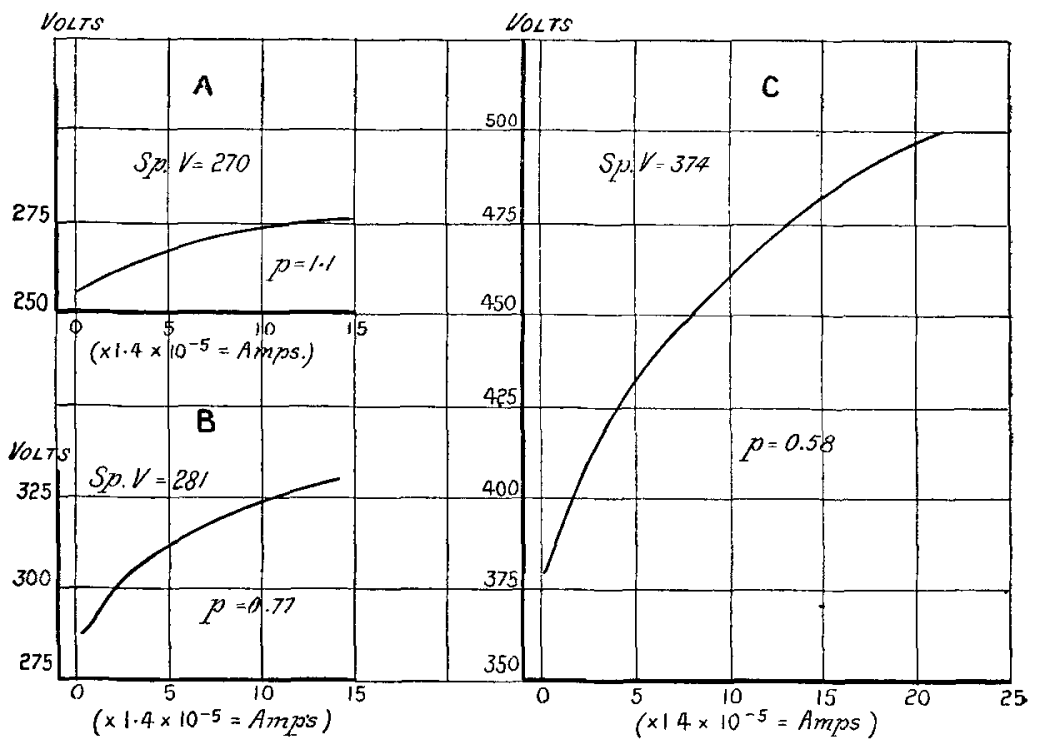

Below the Critical Pressure.

minimum sparking potential. Here there is a slight drop from the sparking potential to the potential required to maintain the current; but it soon rises until it passes the sparking potential and remains above it as far as the investigation was carried. At lower pressures this is still more easily seen. Curves B and C (fig. 4) show absolutely no drop; the curve at first increases very rapidly from the direction of the sparking potential, and gradually seems to approach a limit.

These curves are all the average of a large number of sets, which sometimes differ considerably in their values, so that undue emphasis must not be put on the absolute values. A sufficient number were taken, howerer, to ensure a fair degree 
to maintain a Current in a Gas at Low Pressures.

of accuracy. Curve $C$, for instance, being an average of eight separate curves, the greatest variation from the mean being 4 per cent., the error for the most part being very much less, and in none of the other curres does it rise to more than 2 per cent. In all cases the general form for the same pressure was the same, the only difference being in the steepness of the curve. Fig. 2 (see above) shows the average number of readings taken for each set, and may serve to indicate the regular nature of all curves obtained.

Thus we clearly see that there are two distinct cases depending on the pressure. Above the critical pressure, the potential to maintain the current drops below the sparking potential, and tends towards a limiting value which may perbaps be independent of the current. Below the critical pressure, the maintaining potential rises more or less rapidly above the sparking potential.

\section{Discussion of Results.}

The first condition of affairs observed, that above the critical pressure, falls in exactly with Professor Townsend's theories previously advanced. Sparking will take place when

$$
\alpha-\beta e^{(\alpha-\beta) d}=0 ;
$$

where $\alpha$ is the number of the new ions produced by a negative ion in going across a centimetre of the gas, $\beta$ is the corresponding number produced by a positive ion, and $d$ is the distance apart of the electrodes.

Knowing $\alpha$ and $\beta$, the sparking potential $\mathrm{X} d$ can then be calculated for a given pressure. This has been found to be in accurate agreement compared with the sparking potential determined experimentally*. The sparking potential determined experimentally may consequently be used in the determination of $\alpha$ and $\beta$. The quantity $\alpha$ has been already determined over a large range of pressures and forces. The same has not yet, however, been done for $\beta$. It has been shown that the value of $\frac{\alpha}{p}$ bears a constant relation to $\frac{\mathrm{X}}{p}$, where $\mathrm{X}$ is the electric force and $p$ is the pressure; consequently from the curve connecting these two ratios the value

* J. S. Townsend, Phil. Mag. vi. p. 598 (1908). 
of a can easily be read. Such a curve is given in fig. $5 \mathrm{~A}$, this being based on the first determination of $\alpha$ made by Professor Townsend*. More recently, for low pressures, when the effect of $\beta$ must be more strongly felt, it was found that Curve B more nearly expresses the true state of affairs.

Fig. 5.

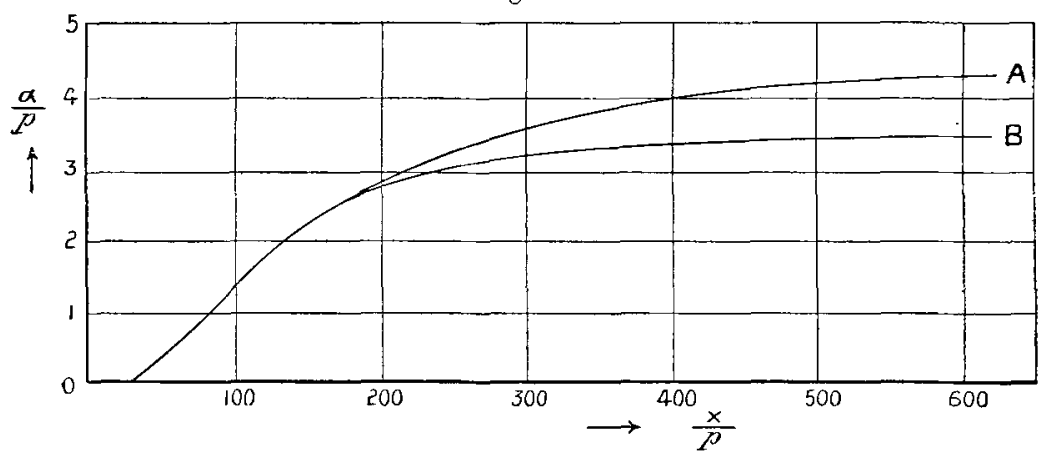

Consequently for the larger values of $\frac{\alpha}{p}$, the quantity read off from this may be taken to be more nearly correct, based as it is on results from the simultaneous determination of $\alpha$ and $\beta$, which Professor Townsend points out as being obviously the most accurate. But it must be remembered that the curve is only an approximation, better determinations being necessary before it can be definitely given.

Taking $\alpha$ then as given by this curre, $\beta$ can be calculated from the equation

$$
\alpha-\beta e^{(a-\beta) d}=0,
$$

and the effects of the positive ions at high pressures and small forces can be determined and compared with the curves previously found, connecting $\frac{\beta}{p}$ and $\frac{\alpha}{p}$. This has been done with two of the curves given in fig. 4 , the values of a being taken as 2.54 and 2.09 respectively for the two pressures $0.77 \mathrm{~mm}$. and $0.58 \mathrm{~mm}$.

A very simple graphical solution gives an easy way of maling this calculation. The above equation may be written

$$
\alpha d e^{-\alpha u l}=\beta c l e^{-\beta d} \text {. }
$$

Constructing the graphs of each of the members of this equation to solve for values of $\alpha d$ and $\beta d$ respectively, they

* J. S. Townsend, Phil. Mag. v. p. 389 (1903). 
will absolutely coincide, giving two solutions for each value of the ordinate. The quantity $\beta$ is known to be small in comparison with $\alpha$ from other work; consequently for each large value of $\alpha d$ the corresponding value of $\beta d$ can be read off directly. Such a curve is given in fig. 6 .

Fig. 6.

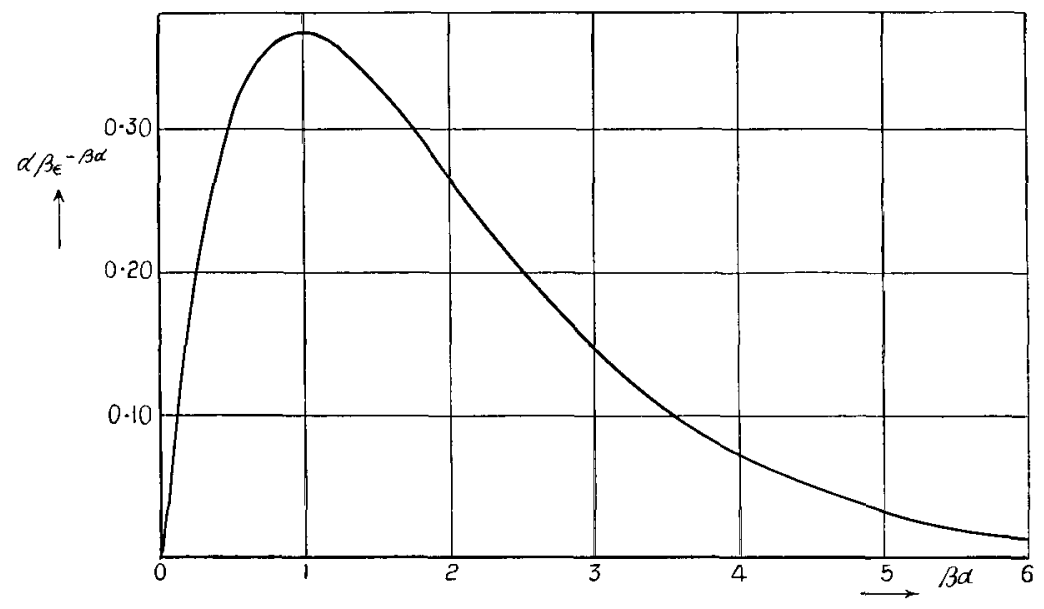

It should be noted, however, that a small error in the determination of the sparking potential will make a very large variation in the value of $\beta$, since the part of the curve where $\frac{\beta}{p}$ is read off is so much steeper than the corresponding part for $\frac{\alpha}{p}$. So this is not a good way to determine $\beta$. Notwithstanding this fact, the results obtained seem to fall in extremely well with the theory. In fig. 7 (p. 220) the heavy line gives the curves showing the relation of $\frac{\beta}{p}$ and $\frac{\mathrm{X}}{p}$ for hydrogen, as determined by Professor Townsend. The two marked points are the values determined for the two curves under discussion, B and C, fig. 4 ; and the dotted line shows how closely they coincide with a continuation of previous observation. The calculation is given in Table $\mathrm{I}$. But it should be noted that in the case of the larger values for $\beta$, and probably at the higher pressure too, it is very doubtful if the equation from which these values have been derived can be expected to hold very accurately. The assumption upon which it is based is that there is a large number of collisions between the ions and the neutral molecules of the gas. But at 
Fig. 7 .

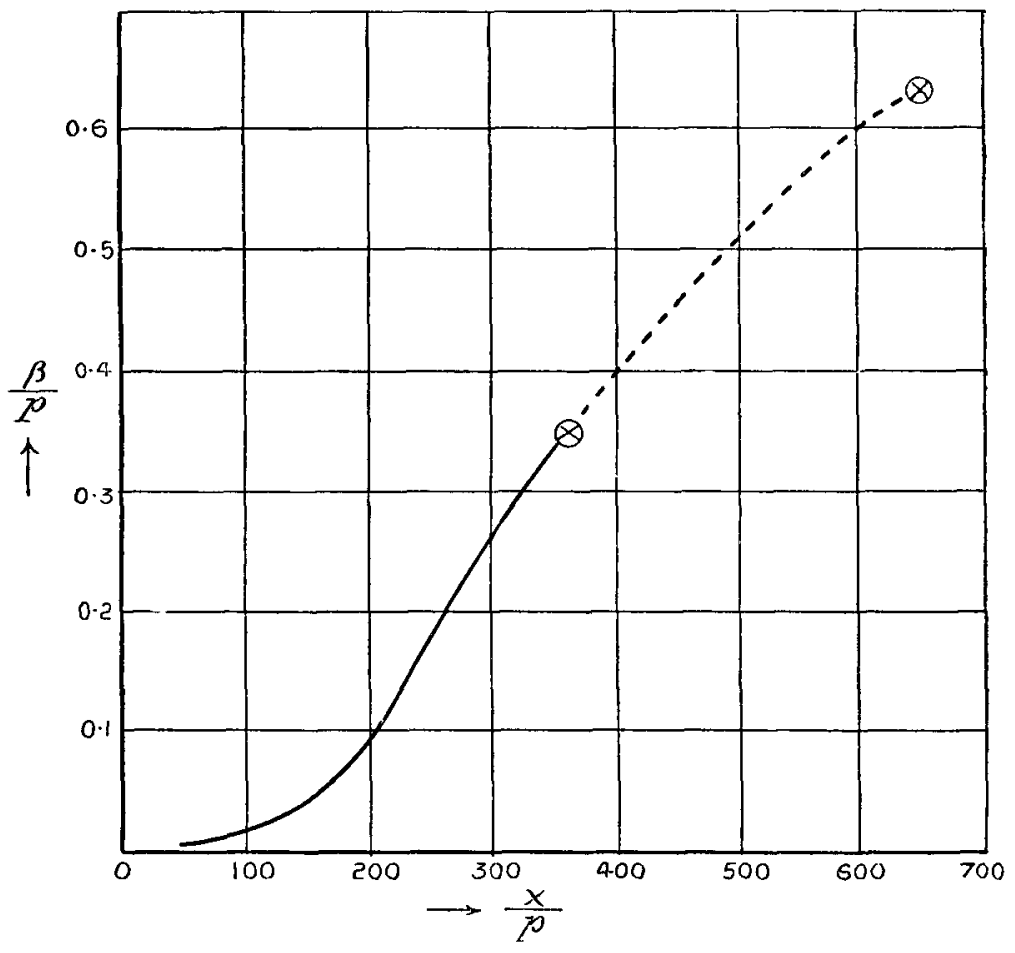

Table I.

Determination of $\frac{\beta}{p}$.

$$
d=1 \text {. }
$$

$$
\begin{aligned}
& p=0.77 \mathrm{~mm} . \quad \text { Sp. V. }=281 \text { volts. } \\
& \therefore \frac{\mathrm{X}}{p}=365 \text {. } \\
& \stackrel{\alpha}{-}=3 \cdot 5 \text {. } \\
& \therefore \quad \alpha=2 \cdot 54 \text {. } \\
& \beta=0 \cdot 26 \text {. }
\end{aligned}
$$

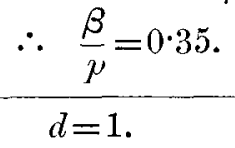$$
\begin{aligned}
p & =0.58 \mathrm{~mm} . & \text { Sp. V. } & =374 \\
\frac{\mathrm{X}}{p} & =645 . & \frac{\alpha}{p} & =3 \cdot 6 . \\
\alpha & =2.09 . & \beta & =0.37 .
\end{aligned}
$$$$
\therefore \frac{\beta}{p}=0.638 .
$$ 
the pressure of only 0.5 millimetre, the mean free path of the positive ions on the kinetic theory, assuming that they are atomic in size, is nearly 4 millimetre, and that of the negative ion about 1.6 millimetre, which is not very small in comparison with the total distance between the plates. For higher pressures when the value of $\beta$ is very small, the determination of $\beta$ in this way must necessarily be more accurate; but in the range under investigation it seems very doubtful whether absolute confidence can be placed in this equation as expressing the true state of affairs, even though it appears to fit in remarkably well with the facts. It may be that the assumed curve for $\frac{\alpha}{p}$ was too low, and consequently the results are more favourable than theoretical grounds would warrant.

Leaving the sparking potential, and coming to the values of the potential required to maintain a current, in the first place, above the critical pressure, a complete discussion of results similar to those obtained in the present work has been given by Professor Townsend *. IIe clearly shows that the difference in the relocities of the positive and the negative ions, tending to cause a surplus positive charge in the gas near the electrode, will fully account for the observed difference between the sparking potential (which is really the potential required to maintain a minute current) and the corresponding potential to maintain larger currents. The latter should be lower, at least for pressures that are above the critical value. Thus the curves obtained (fig. 3 ) fit in exactly with the theory, and lend further confirmation to the previous work.

Below the critical pressure, however, some other explanation of the effect observed must be given. It seems probable that it is masked by another and more marked effect which was not got rid of in the course of the experiments. At high pressures the mass of the carriers is fairly large, the ions being composed of groups of molecules travelling together with a comparatively small velocity. At low pressures and high forces, on the other hand, such as are obtained below the critical pressure, the mass may be very much smaller, in all probability of the order of the mass of a molecule. Hence the velocity is probably very much greater than the velocity at the higher pressures. The charge in the gas for a given current would then be very much less than is obtained with the same current above the critical pressure. We should therefore expect that in this case the drop from the sparking * J. S. Townsend, Phil. Mag. ix. p. 289 (March 1905). 
potential to the potential required to maintain the current would be very small even if nothing else entered to affect the results. The curves found show, however, that there is such a disturbing effect, and the cause is not difficult to see.

The heat energy generated by the current must be considerable, and the presumption is that it will in some way show itself in the action of the discharge. The cause for the noticeable rise of the potential with the current, must then be sought in temperature changes. A direct calculation of the amount of heat energy given to the ions by the passage of the current, in itself is difficult to make on account of the many disturbing influences which tend to lower the temperature. But the rise must be considerable, and, in the course of the present experiments, must have had a modifying influence on the conditions of the gas. Some of the gas must have been driven out through the openings to the sparkchamber, so decreasing the amount of gas between the plates, and consequently raising the potential required to maintain the current. Owing to the sinall mass of the carriers, they must bave given up their heat immediately on striking the massive metal electrodes, and the temperature of the whole mass must have adjusted itself almost at once to the original conditions, the change probably not taking more than a fraction of a second. It will be interesting to test this hypotbesis further, and an experiment is being planned to throw more light on the subject.

On this view, then, the current should heat the gas, driving some of it out of the chamber-the greater the current, the greater the amount of heating, and consequently the smaller the amount of gas left between the plates. But below the critical pressure the smaller the amount of gas, the higher is the potential required to spark, and hence to maintain a constant current. Therefore just as the curves (fig. 4) show the actual state of affairs to be, the potential would rise as the current was increased.

At the higher pressures this effect would not be so noticeable, the driving out of the gas in this case tending to lower the sparking potential, and consequently also the potential required to maintain the current, the effect being in the same direction as that caused by the polarization or charge in the gas spoken of before.

Miention must be made of the explanation given by Stark * in the new edition of Winkelmann's Handluch der Physik, of similar results obtained by Riecke and others under much

* J. Stark, Winkelmann's Handbuch dor Physik, ir. 1. p. 518. 
less definite or determined conditions. He explains the observed phenomena by saying that the nature of the curve is determined by the predominance in the tube of one or another of the characteristic parts that are associated with the discharge through gases at low pressures. In the "dark space," when the current increases, the potential rises, the same effect being observed in the space of the "cathode fall of potential," provided that this is no longer normal, $i$. $e$. that the glow covers the whole surface of the cathode, so that it cannot expand any further. Before that, the cathode fall is independent of the current as well as of the pressure. In the "positive column," on the other hand, the potential decreases with a growing current. A perfectly general curve, connecting the current and the potential required to maintain it, would be given by fig. 8 . Starting at small

Fig. 8.

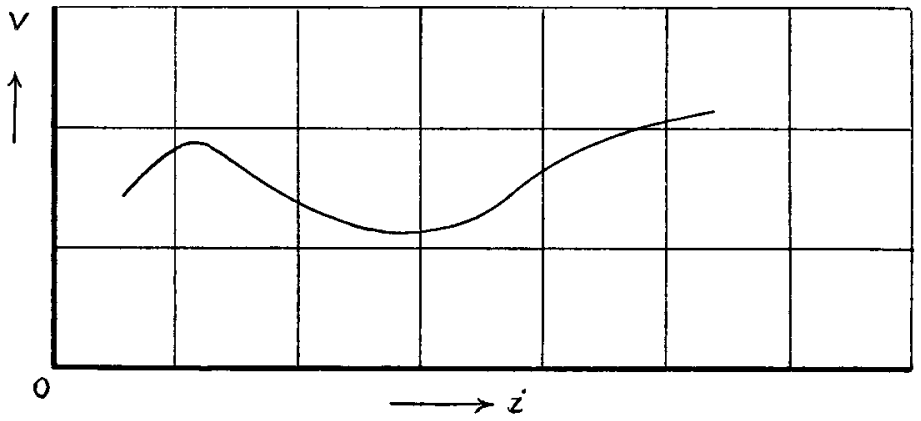

currents, the potential would at first rise with the current, the dark space having the preponderating influence. Reaching a maximum, the pusitive column would begin to encroach upon the dark space until it would overbalance it, and the potential would begin to fall. Hitherto the cathode fall has been constant, but it now begins to rise, so that the potential goes up again. Not all of these conditions, he declares, will be observed at a given pressure. For high pressures the dark space will predominate; for smaller pressures, but above one millimetre, the second characteristic part of the curve will be observed, while finally, for very low pressures, the cathode fall would alone determine the nature of the curve.

This representation, which is framed to account for the observed tacts, cannot be said to be entirely satisfactory. The cathode fall has been shown to equal the minimum sparking potential, so that at the critical pressure the cathode 
fall is exactly the same as the difference of potential between the electrodes. So when the pressure is decreased below this value, the actual cathode fall as such has ceased to exist. In the present investigation with a sparking distance of one centimetre, the critical pressure was about $1.1 \mathrm{~mm}$.; so that in all the cases noted, when the potential rose with the current (fig. 4), the actual cathode fall of potential was no longer anything detinite. On the other hand, it is most improbable that there should not be a considerable heating effect under the circumstances, which would of necessity cause the gas to expand and drive some of it out from between the plates. That being so in any case, it is much simpler to find in this the explanation for the phenomenon without calling in further hypotheses.

Thus, to sun up the explanations given to the observed conditions :- In Case I., where there was a drop from the sparking potential tending toward a constant value, a polarization effect is set up in the gas, which tends to maintain the current at a lower potential than is needed to cause the current to pass originally. In Case II., below the minimum sparking potential, when the potential to maintain the current rises with the current, the polarization is small, and the rise in the potential is to a large extent due to a heating effect which drives out some of the gas.

\section{PART II.}

\section{Some Peculiurities of the Spark-Discharge.}

It has seemed worth while to tabulate a few of the peculiarities noticed at various times during the course of the investigations, some of which entered as sources of error which had to be eliminated or which disappeared of themselves, and others which did not seem to affect the results given, and to which in consequence less attention was paid.

\section{A. Effect of the E.M.F. of Battery on I'alue of Potential required to maintuin a given Current.}

As was mentioned above, the curves at first obtained were often very complicated, especially at high pressures, when one would expect, from previous work, that the curve would be practically straight. The variations in the current were produced in this case by changing both the resistance and the number of the cells in the battery. On going over the Tables, it was found that whenever there was a small resistance 
in the main circuit, the potential-difference at the electrodes in the gas would be high. Series of readings were accordingly taken for values of the potential at the electrodes when

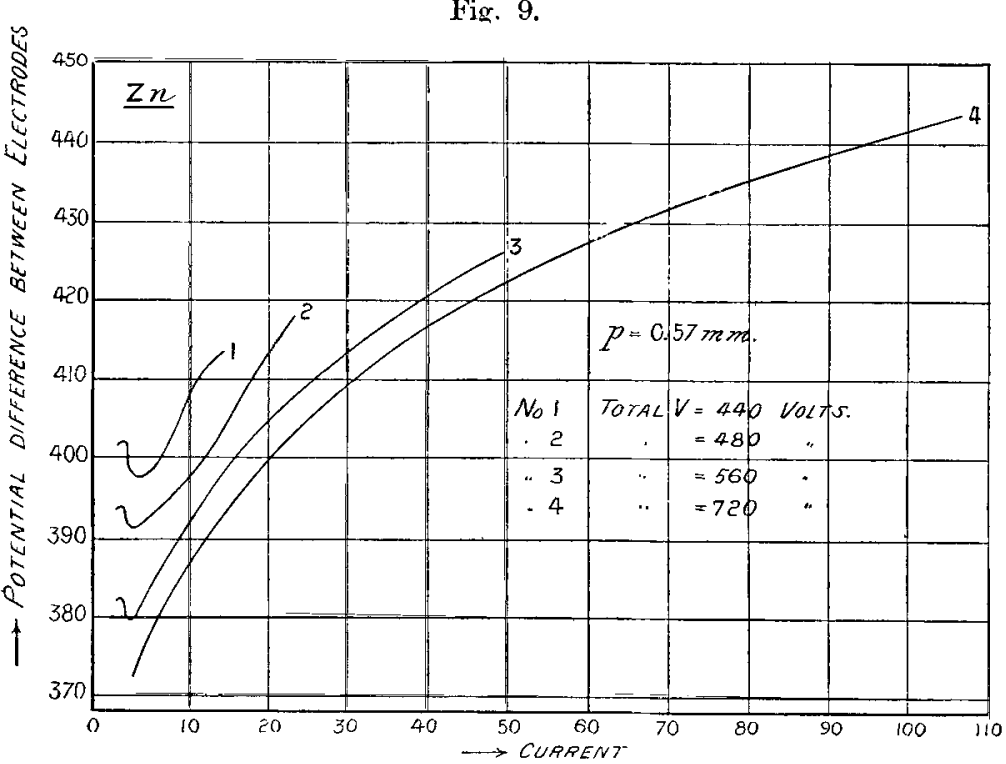

Fig. 10.

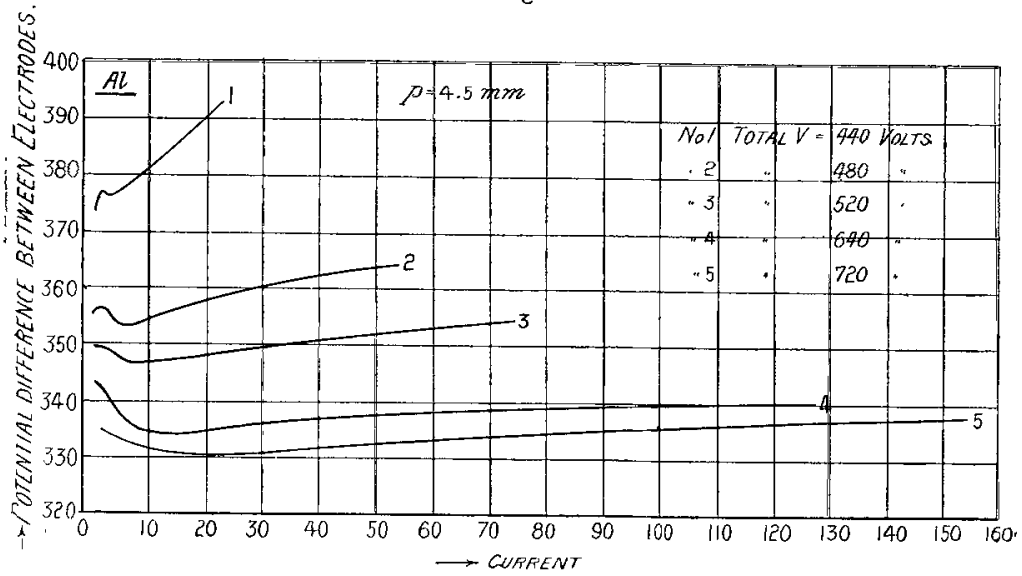

the number of the cells was kept constant and the current altered by changing the resistance of the circuit, a number of curves for different forces being obtained.

Two sets are given, figs. 9 and 10, the E.M.F. of the cells Phil. May. S. 6. Vol. 12. No. 69. Sept. 1906. 
used being indicated in each case. One set was taken above and one below the critical pressure, which, with the sparking distance used, through the whole course of the investigation was always approximately at $1 \cdot 1$ millimetre. In both cases the potential to maintain a definite current will be seen to be lower as the number of cells is increased. The drop is not a constant one however, as the lines tend to come closer with fairly large total potentials, very little decrease being noticed when the number of cells is increased beyond that indicated by Curve 5 (fig. 10). From a priori reasoning it would seem to follow that when external resistance and electromotive force are large, the potential of the electrodes should attain a definite value. This has accordingly been taken as being nearest the true value for a steady current, as it is obvious that a current is most stable when both $R$ and $E$ are large.

This explains the reason why the sets of curves in Part I. of this investigation were taken in the way that they were, i. e. keeping a large force on (1040 to 1200 volts), and varying the current by changing the ohmic resistance. It should be mentioned, in passing, that the sparking distance of the zinc and the aluminium was not exactly the same length, a fact that was only to be expected from the way in which the gaps were put together. This will explain any little discrepancy between tables taken with different metals during the latter course of the experiment (but see below).

\section{B. Effect of Material of Electrodes.}

When the apparatus was first planned it was assumed, on the evidence of. previous investigators, that the material of the electrodes would have no effect on the sparking potential, and consequently on the potential required to maintain the current. The electrodes were accordingly made of zine, as it happened to be the most convenient metal on hand. It was found, however, that the zine tended to form a film on the glass walls of the sparking-chamber, the spark-length in this case being only 0.5 centimetre, and so to give a metallic path for the current. It did not, however, make a stable connexion, so that definite determinations for permanent correction could not be taken as at first hoped. It was in trying to obviate this that the effect on the sparking potential due to the electrodes was first noticed.

Another gap with aluminium electrodes was put in to do away with the disintegration observed with the zinc. It was soon noticed, however, that the two sets of electrodes did not behave in the same way. After a fairly large current 
to maintain a Current in a G'as at Low Pressures.

( $\frac{1}{2} \frac{1}{0}$ ampere) had been running for some time, it was found that the sparking potential was considerably changed. It was at first supposed that the gas was being heated up and driven out from between the electrodes. A thermometer, however, placed in close contact with the plates showed not the slightest increase in temperature, and further, all the gas being pumped out to $\frac{1}{80}$ of a millimetre, and fresh gas adnitted and adjusted to the same pressure as before, seemed to have no effect on bringing back the sparking potential to its original value. If the plates were left to themselves the sparking potential would gradually return, after about twentyfour hours, to what it was before the current passed. But the most peculiar thing was that while for zinc, running the current raised the sparking potential, for aluminium it lowered it. The following Table shows this effect:-

\section{Table II.}

$$
\begin{gathered}
\text { Effect of Current on Sparking Potential. } \\
p=45 / 80 \mathrm{~mm} . \\
\text { Sp. V. . Al }=436 . \quad \mathrm{Zn}=380 .
\end{gathered}
$$

Current (1/4000 amp.) run for $15 \mathrm{~min}$. between $\mathrm{Al}$ electrodes. $p=47 / 80$, adjusted to $45 / 80$.

Sp. V. .. Al $=392 . \quad \mathrm{Zn}=362 . \quad \mathrm{Ag}=442$.

Allowed to stand 3 hrs. $p$ then $=45 / 80$.

$$
\text { Sp. V. . Al }=422 . \quad \mathrm{Zn}=3 \text { б̋. } \quad \mathrm{Ag}=438 .
$$

Current run for $15 \mathrm{~min}$. between $\mathrm{Zn}$ electrodes. $p=47 / 80$ adjusted to $45 / 80$.

Sp. V. .. Al $=446 . \quad \mathrm{Zn}=472 . \quad \mathrm{Ag}=448$.

Allowed to stand $20 \mathrm{~min}$.

Sp. V. . Al $=448 . \quad \mathrm{Zn}=458 . \quad \mathrm{Ag}=454$.

Allowed to stand $20 \mathrm{~min}$.

Sp. V. . Al $=446 . \quad \mathrm{Zn}=44 \delta . \quad \mathrm{Ag}=454$.

It will be noticed that ths values of the sparling potentials for all the spark-gaps seems to change in the same direction, an effect undoubtedly due to a change in pressure in all the gaps; as it is difficult to readjust the pressure instantly, and small variations make large cbanges in the sparking potentials, it cannot be expected that the other spark potentials should return exactly to their previous values. It will be seen, however, that after running the current between the aluminium plates, the decrease in the sparking potential for these is much greater than for the other two sets, when the current had not been flowing; while the same is true for the marked increase

Q2 
in value for the zinc, nearly six times larger than the increase of the other two.

The holes in the plates were quite large enough to ensure an immediate adjustment of pressure in all these gaps, which were joined in parallel. The fact that the gange showed an increase in both cases, shows that we are not dealing with an absorption of gas in the one case and an emission in the other, as one might a priori be led to believe. That this cannot be an effect in the gas is thus definitely shown from the fact that the two metals had an opposite effect on the sparking potential, and that pumping out and admitting fresh gas (not shown in Table) did not influence the process of return to its original value. Many sets of this kind were taken, with several different spark-gaps, the last ones being always about one centimetre in length, and all with consistent results; but it should be noted that at higher pressures, above the critical pressure, this effect was much less noticeable. Two plates of absolutely pure silver were obtained from Messrs. Johnson, Matthey \& Co., of London, and were found to behave exactly as the zinc, $i . e$. running the current raised the sparking potential.

It was not absolutely certain, however, that the apparatus was entirely air-tight, and it was feared that some oxygen or other gas might be having a temporary chemical effect on the electrodes. Great care was accordingly taken to obviate any possibility of leak, and the apparatus was left standing with only hydrogen in contact with the plates for about a month. On resuming work again, it was found that this effect had entirely disappeared; nor after two months' constant use has it made its appearance again. It should be said that absolutely no air has been allowed to come in contact with the plates since that time. Hydrogen has been the only gas having access to them. It seems probable that occluded gas has something to do with this effect, which is undoubtediy on the electrodes and not in the gas of the sparking-chamber.

Whatever its cause or seat, it has certainly disappeared with the assurance of the presence of no gas but hydrogen. Since then, the only difference between the results obtained from the aluminium and the zinc electrodes has been the slight and consistent one already mentioned, due probably to a small difference in the distance apart of the electrodes.

\section{Effect of Reversing the Current.}

Another proof that the effect noted above was the result of something closely connected with the plates, and not a phe- 
nomenon due to a change in the gas, was shown by the behaviour on reversing the current. Table III. shows a typical case.

TABLE III.

Effect on Sparking Potential of Reversing the Current.

$$
\begin{aligned}
\text { Jan. } 25,1906 . \\
\text { Zn. } \\
\text { Sp. V. with At }=391 \text { volts. } \\
\text { " } \quad \mathbf{A}_{-}=403,
\end{aligned}
$$

After ruming current 1/4000 amp. 11 mins.

Sp. V. with $\mathrm{A}+=431$ rolts.

$$
", \quad \mathrm{~A}-=405,
$$

The sparking potential to start with was in general the same whether the top plate (A) was positive or negative. After running the current with A positive, the sparking potential taken in the same way showed an increased value in this direction, if the plates were zinc; but if reversed, i. e. A negative, the sparking potential was still at its original value. This phenomenon must be distinguished from the previous as it is a very temporary one, the effect disappearing almost immediately; and a further difference is that the effect is in the rame direction, whaterer the metal. It has been found that the current running only for a few seconds will have this effect, while the effect due to the material of the electrodes, spoken of before, required a large current for a number of minutes, and lasted for hours after the current had been stopped. Unfortunately, it was not thought of in time to take the sparking potential in the reverse way from which the current had been running, in investigating the previous effect; so this having disappeared, there is no criterion to decide whether the presient phenomenon could also have been distinguished then, when much larger changes in the potential due to running the current were available. In general, the determination of the sparking potential, especially with fresh gas or some that had been left standing overnight, did not immediately give its permanent and correct value. Quite a large force could be put on before the spark would pass, but as soon as this had happened, the proper value for the sparking potential could be obtained; and in the new state of affairs, further sparking would have no appreciable effect on the value. This effect was the same whatever the material or the original direction of the current. On reversing the electrodes, however, the value for the sparking potential was alway, lower, gradually rising to the same value as for 
the original direction. In other words, if, for example, at first the sparking potential was taken with the top plate (A) positive, and then the poles reversed, and without running the current further the sparking potential determined with $\mathrm{A}$ negative, its value now would be considerably lower than it was at first with A positive. Running the current with A negative, however, would bring the sparking potential up to the same value as that obtained originally for $\mathrm{A}$ positive. If now the poles were again reversed without running the current, A positive would in turn be at first lower, gradually rising to its original value. Table IV. shows a typical set of observations which will make this effect clear.

It looks as if there might perhaps be some kind of polarization-effect on the glass walls of the spark-gap, caused possibly by ions adhering to the sides which wonld tend to affect the uniformity of the field between the plates. Whatever the cause, it is an effect that only enters into the determination of the sparking potential, as no similar phenomenon was noticed in the value for the current on reversing the poles.

\section{Other Effects.}

(a) The effect of capacity in the circuit already noticed by previous investigators was also confirmed. When the electrostatic voltmeter was thrown in, the current in general increased, sometimes as much as 10 per cent. This effect was entirely got rid of by having a large resistance in the main circuit. With the series given in Part I. of this investigation, there was not the slightest indication of any error due to this cause.

(b) Another result observed by previous experiment was that the current is not always steady, even though not the slightest flickering in the glow of the spark could be detected, and the galvanometer deflexion remained absolutely stationary. The effect was noticed when a telephone-receiver was put in the circuit. Frequently there would be a shrill whistling noise in the instrument, which would change with the capacity, as when the metallie conductor was touched with the hand. It also varied with the resistance, and scemed quite erratic, as one could never be certain what the pitch of the note would be, nor even if it would be present in a given case. With the large resistance, however, the value of the current seemed entirely independent of the presence or absence of the noise; so in general, except for noting when the phenomenon was present, no attention was paid to it. 


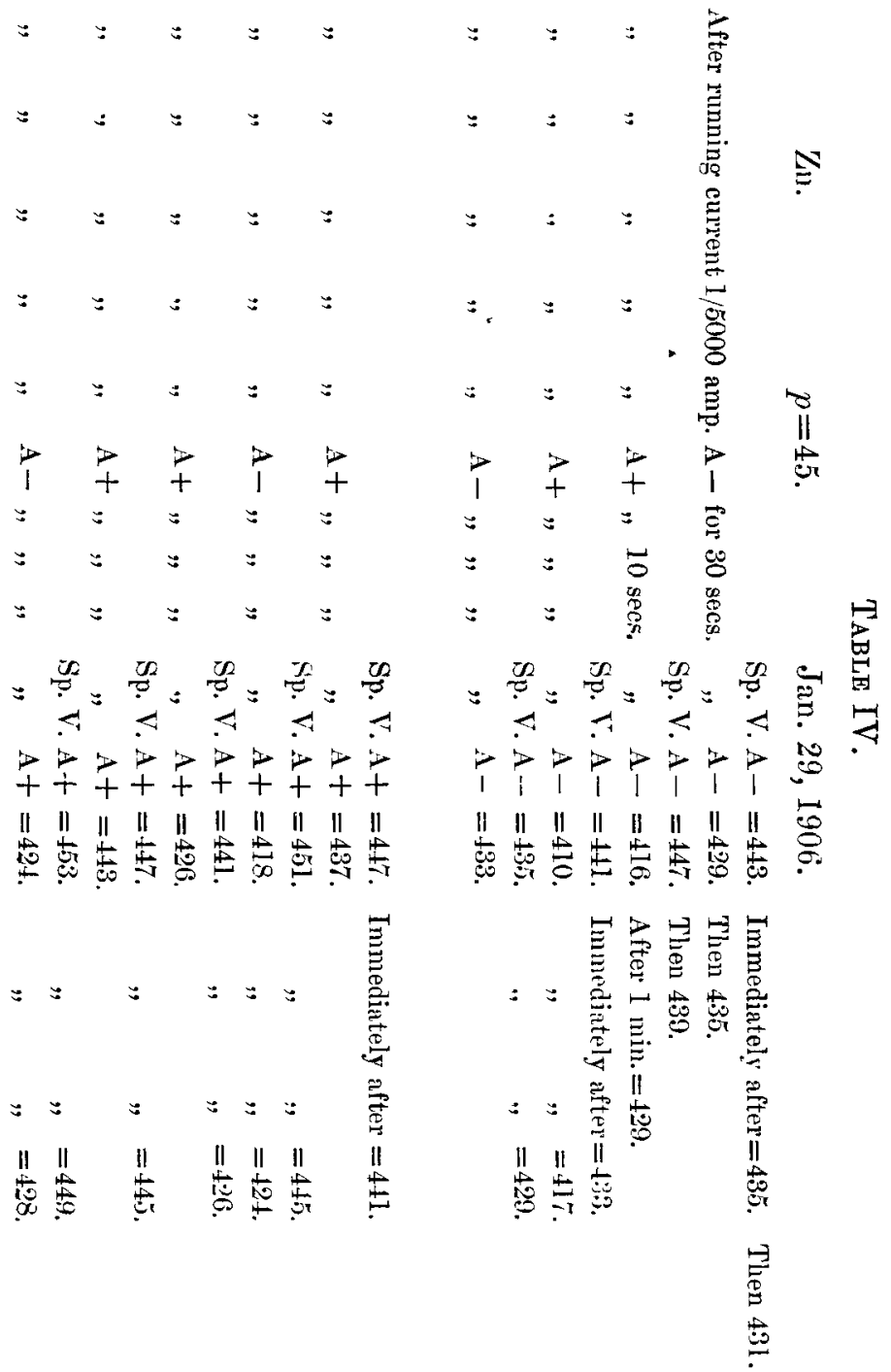

What significance all these effects have, or what modifications not yet eliminated they introduce, it is as yet impossible to say. It is hoped that some further investigation may clear them up. At any rate, with so many peculiarities that are constantly met with in investigations of the spark discharge, it has seemed worth while to make some sort of 
232 Potential required to maintain t Current in a Gus.

tabulation, as above, to indicate some of the difficulties to be met with in work of this kind.

Work is now going on to determine some of these things more definitely, as well as possibly to repeat all the observations with another gas, perhaps nitrogen.

\section{Conclusion.}

To sum up :-

Various peculiarities in the behaviour of a spark-discharge must be noted, which tend to complicate an apparently simple investigation.

(a) A large number of cells is necessary to secure stable conditions.

(b) Occluded gases, or other canses, may tend to introduce subsidiary results depending on the material of the electrodes.

(c) The capacity of the electrodes affects the current when the total external ohmic resistance is small.

(d) The sparking potential is slightly affected by the previous direction of the current, this not being a gas effect, and due either to the sides of the chamber or the electrodes.

(e) The current may be intermittent, even if the appearance of the spark or the galvanometer (not sensitive) shows no unsteadiness.

None of these things, howerer, seemed to vitiate the folJowing main results of the investigation so far established.

(1) The potential required to maintain a current in general varies as the current is increased.

(2) At pressures abore the critical pressure this value is lower than the sparking potential, tending toward a limiting value.

(3) At pressures below the critical pressure it at first rises very rapidly, as the current is increased, the smaller the pressure the more rapid being the rise.

In conclusion, I must express my deep appreciation of the unfailing kindness of Professor J. S. Townsend, under whose direction and constant guidanee the present work was undertaken and carricd out.

University Museum, Oxford. 